\title{
A Brief Discussion on the Development of University Culture Promoted by Photo Archives
}

\author{
Ying Zhang \\ Jilin Agricultural University Archives \\ 474269500@qq.com
}

Keywords: Photo archives; University; Cultural development

\begin{abstract}
The photo file is an important classification of file resources, with the function of information storage and cultural dissemination. Because it is more vivid, cubic and acceptable by the public in terms of the historical records and cultural communication, it plays a unique role in the development of University culture. Take the archives of Jilin Agricultural University as an example, this paper studies the problems existing in the photo archives of colleges and universities, and expounds some suggestions on how to perfect the work of the university's photo archives and promotes the development of the university culture.
\end{abstract}

\section{Introduction}

Photo archives refers to the digital image and social organizations or individuals in social activities formed directly in still photographic image or by scanner, digital camera direct shooting was mainly reflects the historical record has kept the value of the. In the archives of colleges and universities, the photo archives is an important part, with its unique carrier form, reflecting the development of the school, the major historical changes, important events and the face of the characters.[1] The photo archives play an important role in the compilation of history, the compilation of characters, moral education and publicity. The developed countries began to pay attention to the role of archives on cultural development from 1920s, our country from 1980s gradually pay attention to the cultural function of the archives, with the continuous development of the cause of the school archives (room) to invest a lot of manpower and material resources to the photo archives were collected for development and utilization. Jilin Agricultural University archives, for example, I have a library of about 22400 photos, from the beginning of the school in 1948 only 11 photos to the 2016 of the more than and 300 photos, these photos bear the development and heritage of our school. These photos not only make people understand the school's long history, but also give people a vivid impression.

The cultural communication function of the photo archives makes the utilization degree and the attention degree of the photo archives more and more high. However, the work of the photo archives is still lagging behind, and the filing system and management procedure of the photo archives are still not perfect. In a sense, it restricts the pace of the development of the University culture. Based on the actual work, the author puts forward the following points.

\section{The Characteristics of Photo Archives and Its Significance to the Development of University Culture}

The photo archives can play the role of historical evidence, is to preserve the value of the historical records, the photo archives has its own characteristics: original records, visual image, supplementary proof, extensive communication, carrier diversity, not regenerative.[2]Photo files can be divided into traditional photo archives and digital photo archives. Traditional photo archives is a kind of historical record with the value of preservation as the carrier and the image as the reflection mode. It consists of negatives, photographs and illustrations. Digital photo archives mainly from two aspects: one is a digital video recording equipment filmed by digital technology, two is the traditional picture, audio and video files (via computers, scanners and other equipment) of the. Digital photo is the electronic document, has the following advantages: easy formation, 
intuitive reading, memory mass storage, intelligent operation processing, reduce the stability and rapid information copy volume. Collection photo archives of Jilin Agricultural University, because of the age, in the consolidation, basically in the traditional picture, in person after finishing, after identification, scanning into highly clear after the formation of the electronic version of the photo archives electronic photos, and recorded in the archives management system.

Because of the photo archives directly and vividly reflect the objective things and the surrounding environment exists, and intuitive, contains abundant information, make people the wealth of information obtained from the real image so that the widespread use of various units, so do the collection, management and use of photo archives in Colleges and universities, to standardize the management of files, promotion has important practical significance of university culture development.

\section{The Main Problems Faced by University Photo Files}

With the development of global information in recent years, especially the emergence and rapid development of new media, the archives management of colleges and universities has entered a new historical period. However, there are many problems in the work of photo archives in colleges and universities, such as the dispersion of photos, the diversity of methods, the uneven quality of photos, the difficulty of fidelity, confidentiality and preservation. Embodied in the following aspects:

The Overall Weak Awareness. This kind of archives consciousness reflects the importance and cognition of archives in Colleges and universities. Original photo archives started relatively lag, plus the picture files in universities started late, campus activities is diversified in recent years, the understanding of the photo archives is far less than the file on the digital photo shoot, awareness of the collection and preservation of old ideas, archive consciousness, generally considered the photo archives of no great importance. Some institutions or individuals because the job is busy, so pay attention to the photo archives accumulation, no special custody or even long scattered storage because the managers job changes caused by the photo no handover, stored or discarded piles. The photo archives collection due to a wide range of management personnel changes, some units or staff do things carelessly, for the convenient use of units and individuals, will find all kinds of excuses not to file, a serious lack of awareness of archives and so on. These factors have seriously affected the collection, collation and compilation of photo archives.

The Non-Perfect Management System of Photo Archives. According to the national standard "and" photo archives management "in the electronic filing management measures" provisions, although many colleges and universities to develop the system of photo archives management, but also is inadequate, resulting in the actual work in the collection, custody of the photo archives and use are not specific and not standardized, and therefore can not form an organic overall, unsystematic.[3] The management department of the file management, the supervision and guidance of the photo file is also lack of direct and specific, which will directly affect the management and utilization of photo files.

The Fragmented Photo Archives. Because the university personnel constraints, many archives (room) to send someone to follow school major events, important meetings and other important events, including the student activities has an important preservation value. These photos or by the University publicity department or units directly involved in the shooting, so that a more diversified picture phenomenon obviously, making the number of photo files, although increasing the sources increased, but the units and individuals involved in filing and archiving the dispersion, this person consciousness is not strong, it will cause difficulties, collect scattered sources of photo archives the phenomenon of.[4]

The Untimely Filing of the Photo-Missing Phenomenon. Digital photos only scattered stored on a personal computer, save only by a simple folder, file managers only a little finishing, not by screening the entire over to the archives (room), on each photo captions and annotations are not specific, and finishing is not timely the serious situation. In addition to form and store digital photos mainly rely on modern computer equipment, video equipment and storage equipment, will directly 
affect the safety of the digital photo hardware condition, and the storage format of renewal, damage and loss of digital photos.

The Condition of the Photo Archive not Complying with the Requirements. At present, lags behind the development of archives work in Colleges and universities, the degree of attention to the archives work is not enough, widespread phenomenon, a serious shortage of funds for archival facilities, office and warehouse to do the "three separate", the Treasury is not up to the requirements of "eight protection", the warehouse temperature and humidity is not up to the standard, photo archives there is no more professional storage equipment, resulting in easy deterioration, damage of photo archives. Jilin agricultural archives, for example, there are two problems: first, there are 149 long photos due to age, has been damaged, unable to scan uploaded to the file management system. Two there are more than and 56 photos only the old version, no photo has no old-fashioned washing technology and medicine, will not wash out the picture.[5]

\section{Perfecting Photo Archives in College to Promote the Development of College Culture}

Strengthen the awareness of the collection of photo archives in Colleges and universities, and the publicity of archives awareness. Timely and regular collection, the authenticity and quality of the photos to be strictly controlled, if you can not take photos together, then the management and utilization of the photo file is simply out of the question. (1) for colleges and universities now for each photo file collection, management awareness of the status quo, the archives (room) to increase the value of the publicity of the photo file, through the campus website, WeChat and micro-blog and other new media to enhance awareness of the photo file.[6] In addition, through exhibitions, compilation album and use effect display method, combined with the "Archives Law" "photo archives management norms" and other laws and regulations, publicity photo archives value to the teachers and students, help them to understand the photo archives in higher education, research and social publicity in the important position and role, expand the influence of photos archives in Colleges and universities, teachers and students get support. In the archives of Jilin Agricultural University as an example, we have collected in 50s and 60s photo editing. (2) the characteristics of archives collection of photographs, can open to the inside and outside of the college, especially the retired veteran comrades, or the late old comrade's family collection of old photographs of my school in this way has collected 86 pieces of precious photos, rich collection photos. (3) to strengthen the tracking of major events in universities. According to the events of the formation of photo archives (room) should also be actively tracking collection, should be involved in the event process when necessary, in order to more clearly picture of the background, to ensure the integrity of the file photo.

To improve the rules and regulations of the photo archives, to formulate the rules for the implementation of the archival filing of the universities, and to ensure the timely filing of the photo archives. To carry out many forms of photo archives collection work, strengthen the standardization management of University Photo archives. At present, most of the University Archives developed a number of regulations, but in comparison, the photo archives system is not perfect, operability is not strong, to guide the practical work is not strong, rather than teaching, administrative and party documents detailed and feasible etc.. Photo archive photo archives filing rules for the implementation of response time, picture archiving scope, background information notes, storage conditions have made detailed provisions, the focus is on file specification make a detailed description of the photo archives.[7] Our school to carry out scientific and effective management of archives, archives according to the "People's Republic of China law" and "ordinary college archives management measures", combined with the actual school archives work, formulated the "Interim Provisions" and "Jilin Agricultural University Jilin Agricultural University archives management documents filing".

In accordance with the principle of "centralized selection, storage, sees each other, long-term preservation", it's better to timely collect photo archives in Museum collection. This is related to the use of the future, but also the basis for the work of photo file image editing. This includes two aspects, one is the formation of the photo archives to the photo department in time, as with the hand, 
especially to fill out the photo archives, so as not to forget, cause unnecessary trouble, that indicated on the figures, time, places and events, background, must be detailed and complete, to straight text short and clear. Two to maintain the integrity of the photo archives, filled the missing items, only the electronic version of the photo to flush out the photo paper; only paper photographs should be scanned into electronic version of high resolution. Three for the collection of photos of the file is not easy; the archives (room) must cherish, and into the screening, pick out the theme of bright, clear picture, color saturation of the photo finishing.

Compile and develop the photo archives, promote the development of the university culture and serve the society. Photo archives is an important witness of historical development, we can intuitively understand that images of life in historical period, a literature attribute cannot be replaced and indispensable, has a unique historical value, is extremely precious cultural heritage. Because of its vivid image and strong sense of time and space, the photo archives are popular with the public. The conditional archives should set up the photo archives database as soon as possible, and expand the scope of the use of the photo archives.

Taking Jilin Agricultural University as an example, the 22400 photo archives content involves four main direction of school change, school construction and development of teaching and research, and campus culture, we follow the chronological order of events and time, every 10 years for a photo album than. Every year, the order of the photos is: school change, school development, teaching and research and campus culture. (1) the order of the change of the school is the school gate, the vision, the teaching building, the student dormitory and the staff quarters. (2) the order of the construction and development of the school is: Leadership inspection, cooperation and exchange, organization construction. (3) the order of teaching and research is: Teachers' teaching research, students' teaching practice, teaching facilities. (4) the order of campus culture is: sports activities, cultural activities, social activities, graduation photo. Each series of photos in accordance with the sequence of events. All the old photos are kept as before, did not make any modifications, will be filled with dust in the past to show the world, while marking the file number, easy to query and use.

\section{Conclusion}

The photo archives is of great significance in promoting the development of university culture, colleges and universities in the photo archives promote the cultural construction of universities is still a long distance, we actively strengthen the propaganda of the photo archives work, promote the establishment of the system of the collection, management and utilization of the photo archives, photo archives to speed up the pace of information technology, and actively carry out the development and utilization of increase the photo archives information resources, provide the impetus for the development of University culture.

\section{References}

[1] J.J Gou, Z.H. Hao. The Simple Interpretation of both the Digital Photo File Management and "Digital Photo Archiving and Management Standards (Draft)" [J]. Managers, 2014 (21)

[2] J.X. He. The Design and Implementation of Digital Photo Management System of Guangxi University of Finance and Economics [D], University of Electronic Science and technology, 2013

[3] D.S. Duan, Photo Archive [M]. Writers Publishing House, 2011

[4] Y.X. Zhang Research on the Protection of Audiovisual Archives from the Perspective of Social Memory [J]. Beijing archives, 2013, (12)

[5] R.F. Dong, On the Importance and Management of the collection of photo archives in Colleges and universities, Journal of Jiaxing University, 2011

[6] H.L. Wu. On the Management of Digital Photo Archives of Grass-roots Units [J]. science, 2013 
[7] J. Yang, M. Zhou. A review of the Research on the Digitalization of Audio Visual Archives of University Archives in the past ten years [J]. Yunnan archives, 2015 